\title{
A Comparison of International Management Guidelines for Hidradenitis Suppurativa
}

\author{
Aleksi J. Hendricks ${ }^{\text {a }}$ Jennifer L. Hsiao ${ }^{b}$ Michelle A. Lowes ${ }^{c}$ Vivian Y. Shi $^{d}$ \\ a University of Arizona College of Medicine, Tucson, AZ, USA; ${ }^{b}$ Division of Dermatology, University of California Los \\ Angeles, Los Angeles, CA, USA; ' ${ }^{C}$ Laboratory for Investigative Dermatology, The Rockefeller University, New York, NY, \\ USA; ${ }^{d}$ Division of Dermatology, Department of Medicine, University of Arizona, Tucson, AZ, USA
}

\section{Keywords}

Hidradenitis suppurativa - Acne inversa - Verneuil's disease · Guidelines · Treatment · Management

\begin{abstract}
Hidradenitis suppurativa (HS) is a chronic inflammatory dermatosis that imparts a significant burden on patients and presents a management challenge for healthcare providers. As attention to this debilitating condition has grown over recent years, our understanding of HS pathogenesis and optimal treatment approaches continues to evolve. Nine HS treatment guidelines developed by various expert organizations have been published, encompassing therapeutic modalities ranging from topical agents to systemic therapies to procedural interventions. These guidelines demonstrate significant overlap in treatment recommendations and have all been published within the last 5 years. Therefore, we aim to compare and synthesize the recommendations of international HS treatment guidelines and to encourage inter-organizational communication for the development of consensus or staggered publication of recommendations for $\mathrm{HS}$ management.

(c) 2019 S. Karger AG, Basel
\end{abstract}

\section{Introduction}

Hidradenitis suppurativa/acne inversa (HS) is a chronic, inflammatory, recurrent, debilitating skin disease of the terminal hair follicle that usually occurs after puberty and presents as painful, deep-seated, inflamed lesions in the apocrine gland-bearing areas of the body, most commonly the axillae, inguinal, and anogenital regions (Dessau definition, 1st International Conference on Hidradenitis suppurativa/Acne inversa, March 30-April 1, 2006, Dessau, Germany) $[1,2]$. The estimated prevalence of HS varies from 0.05 to $4.1 \%$ [3], with a recent study of a heterogeneous population-based sample in the USA showing a prevalence of $0.1 \%$ [4], although true prevalence may be higher due to historic underdiagnosis and delayed time to accurate diagnosis. While the pathogenesis of HS has yet to be fully elucidated, a number of factors have been implicated in the disease process, including follicular occlusion, inherent weakness of the folliculo-pilosebaceous unit, altered immune and inflammatory milieu, and frictional exacerbation $[1,3,5,6]$.

Due to chronic pain, drainage, and malodor associated with HS lesions, this condition imposes a significant bur- karger@karger.com

(C) 2019 S. Karger AG, Basel

www.karger.com/drm

Karger ${ }^{\prime}=$
Vivian Y. Shi, MD

Division of Dermatology, Department of Medicine University of Arizona

7165 N. Pima Canyon Dr., Tucson, AZ 85718 (USA)

E-Mail vshi@email.arizona.edu 
den on patients' quality of life and psychological well-being $[7,8]$. High rates of depression and anxiety have been reported in HS patients (prevalence 42.9 and 3.9\%, respectively) $[9,10]$, as well as feelings of loneliness and stigmatization [11, 12], detrimental effects on sexual health (66.7\%) [13], and a nearly 2.5 -fold higher suicide risk compared to the general population [14]. Individuals with HS experience financial strain due to the cost of treatment and disease interference with work and steady employment [15-17]. Furthermore, HS can present a challenge to practitioners in long-term management of a debilitating and often treatment-refractory disease.

Management of HS involves a multi-pronged approach that may comprise lifestyle modifications such as smoking cessation and weight loss, topical therapies, systemic antibiotics, anti-inflammatory and biologic agents, laser and phototherapy, and procedural interventions such as deroofing and wide local excision [3]. Over recent years, several HS management guidelines have been put forth by expert groups in North America, South America, and Europe. This review aims to synthesize published guidelines for HS treatment and compare international management recommendations in order to provide a comprehensive view of evidence-based HS management approaches, including recent supporting data for each section where available.

\section{Methods}

A comprehensive literature search for published HS management guidelines was conducted in June 2019 using PubMed and EMBASE databases. Search terms included "hidradenitis suppurativa," "acne inversa," "Verneuil's disease," "treatment," "management," "guidelines," "recommendations," "consensus," and "expert." Abstracts were reviewed for relevance. Inclusion criteria were articles in English, publications from groups involving dermatologists, and inclusion of comprehensive HS management guidelines. Articles written in a language other than English and recommendations put forth by non-dermatologist groups were excluded.

\section{Results}

Nine guidelines (including one 2-part publication) meeting the inclusion and exclusion criteria were selected (Table 1, presented in chronological order of publication). These guidelines have been established by working groups and organizations including the British Association of Dermatologists [18], US and Canadian HS Foundations $[19,20]$, HS ALLIANCE (international expert group from Europe and North America) [21], Canadian
Dermatology Association [22, 23], European HS Foundation [24], European Academy of Dermatology and Venereology [25], Swiss consensus group [26], and Brazilian Society of Dermatology [27].

\section{HS Assessment}

All reviewed guidelines advocate for early diagnosis by a dermatologist or healthcare provider with experience in treating HS, and address recommendations for clinical grading and assessing treatment response. Recommendations for HS management across all guidelines endorse a multidisciplinary approach including dermatology, primary care, gynecology, psychiatry, pain management, and surgery. Communication and collaboration between providers across specialties is deemed essential for building a comprehensive management plan for HS patients.

\section{Clinical Grading}

Hurley staging is universally recommended across guidelines as a tool for clinical grading of baseline HS severity $[18,19,21-27]$. Hurley staging classifies HS clinical severity as Hurley stage I for single or multiple abscesses without scarring, Hurley stage II for limited scarring and/ or sinus tracts, and Hurley stage III for extensive scarring and/or sinus tracts [28]. Sartorius score is discussed as an alternative grading system in the European S1 and Swiss guidelines $[25,26]$.

\section{Outcome Measures}

Hidradenitis Suppurativa Clinical Response (HiSCR), defined as a $>50 \%$ reduction in inflammatory nodule count and no increase in tunnel count, is cited by the HS ALLIANCE, Canadian Dermatology Association, Canadian consensus, European HS Foundation, and Brazilian guidelines for assessment of treatment response [21-24, 29]. All except the British, Swiss, and Brazilian guidelines also recommend incorporation of the pain visual analog scale and Dermatology Life Quality Index (DLQI) as patient-reported outcomes [19, 21-25].

\section{Screening for Comorbidities}

Screening for comorbidities is discussed in all except the Canadian Dermatology Association guidelines [18, 19, 21, 23-27]. Common comorbidities cited are cardiovascular disease, diabetes mellitus, metabolic syndrome, autoimmune conditions (especially inflammatory bowel disease and inflammatory arthropathies), polycystic ovary syndrome, depression, and anxiety. Early identification of comorbid conditions is crucial for facilitating referrals and establishing a multidisciplinary care team for HS patients. 
Table 1. Hidradenitis suppurativa management guideline publications

\begin{tabular}{|c|c|c|c|}
\hline Organization & Year & Publication & $\begin{array}{l}\text { In-text } \\
\text { abbreviation }\end{array}$ \\
\hline $\begin{array}{l}\text { European Academy of } \\
\text { Dermatology and Venereology }\end{array}$ & 2015 & $\begin{array}{l}\text { European S1 guideline for the treatment of hidradenitis suppurativa/ } \\
\text { acne inversa [25] }\end{array}$ & European S1 \\
\hline European HS Foundation & 2016 & $\begin{array}{l}\text { Evidence-based approach to the treatment of hidradenitis suppurativa/acne } \\
\text { inversa, based on the European guidelines for hidradenitis suppurativa [24] }\end{array}$ & $\begin{array}{l}\text { European HS } \\
\text { Foundation }\end{array}$ \\
\hline $\begin{array}{l}\text { Canadian Dermatology } \\
\text { Association consensus group }\end{array}$ & 2017 & $\begin{array}{l}\text { Approach to the management of patients with hidradenitis suppurativa: } \\
\text { a consensus document [23] }\end{array}$ & $\begin{array}{l}\text { Canadian } \\
\text { consensus }\end{array}$ \\
\hline $\begin{array}{l}\text { British Association of } \\
\text { Dermatologists }\end{array}$ & 2018 & $\begin{array}{l}\text { British Association of Dermatologists guidelines for the management of } \\
\text { hidradenitis suppurativa (acne inversa) } 2018 \text { [18] }\end{array}$ & British \\
\hline HS ALLIANCE & 2019 & $\begin{array}{l}\text { Hidradenitis suppurativa/acne inversa: a practical framework for } \\
\text { treatment optimization - systematic review and recommendations } \\
\text { from the HS ALLIANCE working group [21] }\end{array}$ & HS ALLIANCE \\
\hline $\begin{array}{l}\text { US and Canadian HS } \\
\text { Foundations }\end{array}$ & 2019 & $\begin{array}{l}\text { North American clinical management guidelines for hidradenitis } \\
\text { suppurativa: a publication from the United States and Canadian } \\
\text { hidradenitis suppurativa foundations } \\
\text { Part I: diagnosis, evaluation, and the use of complementary and } \\
\text { procedural management [19] } \\
\text { Part II: topical, intralesional, and systemic medical management [20] }\end{array}$ & $\begin{array}{l}\text { North } \\
\text { American }\end{array}$ \\
\hline $\begin{array}{l}\text { Brazilian Society of } \\
\text { Dermatology }\end{array}$ & 2019 & $\begin{array}{l}\text { Consensus on the treatment of hidradenitis suppurativa - } \\
\text { Brazilian Society of Dermatology [27] }\end{array}$ & Brazilian \\
\hline
\end{tabular}

\section{Nonpharmacologic Interventions}

Smoking Cessation. Smoking cessation is encouraged across all guidelines as adjuvant therapy for HS, citing increased prevalence of smoking among patients with HS compared to the general population and association of tobacco use with more severe disease [18, 19, 21-27]. Although there is limited evidence that smoking cessation reduces HS disease activity [30], discussion of tobacco cessation should be routinely included in HS patient counseling with referral to smoking cessation services when available and appropriate.

Weight Loss. Weight reduction is also universally recommended across guidelines, as higher BMI has been associated with increased HS severity and contributes to frictional exacerbation [18, 19,21-27]. Weight loss should be encouraged as adjuvant therapy for HS, with regular screening for obesity and metabolic syndrome.

Dietary Modifications. The North American guideline is the only publication to address dietary modifications for HS, although it cites insufficient evidence to recommend avoidance of dairy or brewer's yeast [19].

Wound Care. Discussion of wound care and dressing selection is included in the North American, Canadian consensus, European S1, and Brazilian guidelines [19, 23, $25,27]$. All 4 publications recommend that dressing choice should be customized based on location of the lesion(s) and amount of drainage, and selected based on physician expertise with consideration for cost and patient preference. Dressings should be non-irritating and absorbent to reduce skin surface moisture and limit odor. The Canadian consensus, European S1, and Brazilian guidelines address the lack of HS-specific bandages and highlight the need for studies evaluating dressings and wound care in HS [23, 25, 27].

Mental Health Services. All guidelines emphasize the psychological burden associated with HS and endorse screening for depression and anxiety as a routine component of clinic visits. A multidisciplinary treatment team 
including psychosocial services is recommended by all expert groups [18, 19, 21-27]. The European S1 and Canadian consensus guidelines highlight the lack of specific studies assessing the effect of psychosocial support in HS, but suggest that referral to mental health services be conducted in a standardized manner to allow for future analysis $[23,25]$.

\section{Pain Management}

All reviewed guidelines acknowledge the significant pain burden associated with HS, and the majority of guidelines discuss assessing pain severity in HS patients using a validated patient-reported outcome tool such as a pain visual analog scale or numeric rating scale $[18,19$, 21-25]. While most guidelines mention pain management as adjuvant treatment for HS, few provide suggestions for specific analgesic agents. Among the guidelines that do discuss specific agents for pain management, topical analgesics and non-steroidal anti-inflammatories are recommended as first-line modalities, and opioid use is discussed only for severe pain uncontrolled with first-line therapies. Pain specialists are broadly recommended as part of the multidisciplinary approach to HS management.

Topical Analgesics. Topical lidocaine is cited in the North American and Canadian consensus guidelines as a useful modality for pain management in HS [19, 23]. Canadian consensus guidelines specifically suggest lidocaine $5 \%$ ointment, with diclofenac $1 \%$ gel as an alternative topical analgesic [23].

Non-Steroidal Anti-Inflammatory Drugs (NSAIDs). NSAIDs are discussed in the North American, Canadian consensus, and European S1 guidelines as systemic analgesics for pain management in HS [19, 23, 25]. Specific dosing is not mentioned but should generally follow manufacturer recommendations. Special considerations should be made for patients with abnormal liver and kidney function, and use of over-the-counter medications including NSAIDs should be reviewed at routine visits.

Neuropathic Pain Agents. Gabapentin and pregabalin are recommended by the North American and Canadian consensus guidelines for control of HS-associated neuropathic pain, but specific dosing is not addressed $[19,23]$.

Tramadol. Tramadol is an opioid-like analgesic that binds mu-opioid receptors in the central nervous system and inhibits serotonin and norepinephrine reuptake [31]. The North American guidelines recommend tramadol as a non-opioid alternative for HS pain refractory to topical and over-the-counter analgesics, but specific dosing recommendations are not discussed [19].
Opioids. Opioids are cited in the North American, Canadian consensus, and European S1 guidelines as analgesic agents for severe HS-related pain non-responsive to first-line agents such as topical analgesics and NSAIDs. All 3 guidelines urge judicious prescribing of opioids, restricting use to individualized cases, and involvement of pain specialists for opioid monitoring $[19,23,25]$. The Canadian consensus and European S1 guidelines discuss that there is no clinical evidence supporting the use of opioids in HS [23, 25]. For appropriate cases, the European S1 guidelines list codeine and hydrocodone as preferred opioid agents [25].

\section{Topical/Intralesional Therapies}

Recommendations regarding topical and intralesional therapies in HS management are summarized in Table 2. Across all guidelines, topical therapeutic modalities are generally recommended for mild stages of HS and are of most utility in localized disease or as adjuncts to systemic treatment. Topical therapies aim to minimize follicular plugging (keratolytics), decrease bacterial colonization and associated inflammation (antiseptics and antibiotics), and reduce acute pain and inflammation in isolated lesions (intralesional corticosteroids).

\section{Topical Keratolytics}

Resorcinol. Resorcinol 15\% cream is mentioned for its keratolytic and antiseptic properties in the North American, European S1, Canadian Dermatology Association, Canadian consensus, European HS Foundation, and Brazilian guidelines [20, 22-25, 27], but is not mentioned in the British, HS ALLIANCE or Swiss guidelines. Topical resorcinol administered twice daily during flares has been evaluated in a small case series of Hurley stage I/II patients and is recommended as second-line therapy by the European HS Foundation and Canadian Dermatology Association guidelines [22, 24]. Potential adverse effects of topical resorcinol application include desquamation and irritant contact dermatitis [20, 25]. Of note, resorcinol cream is not freely available in the USA and requires compounding by a specialty pharmacy.

\section{Topical Antiseptics}

Topical antiseptics are recommended in 3 guidelines to minimize bacterial colonization and reduce inflammation. Recommended disinfectants vary, and the North American guidelines note that there is no data comparing the efficacy of topical antiseptic agents [20]. Chlorhexidine, benzoyl peroxide, and zinc pyrithione are cited by the North American guidelines [20], while Swiss recommendations men- 


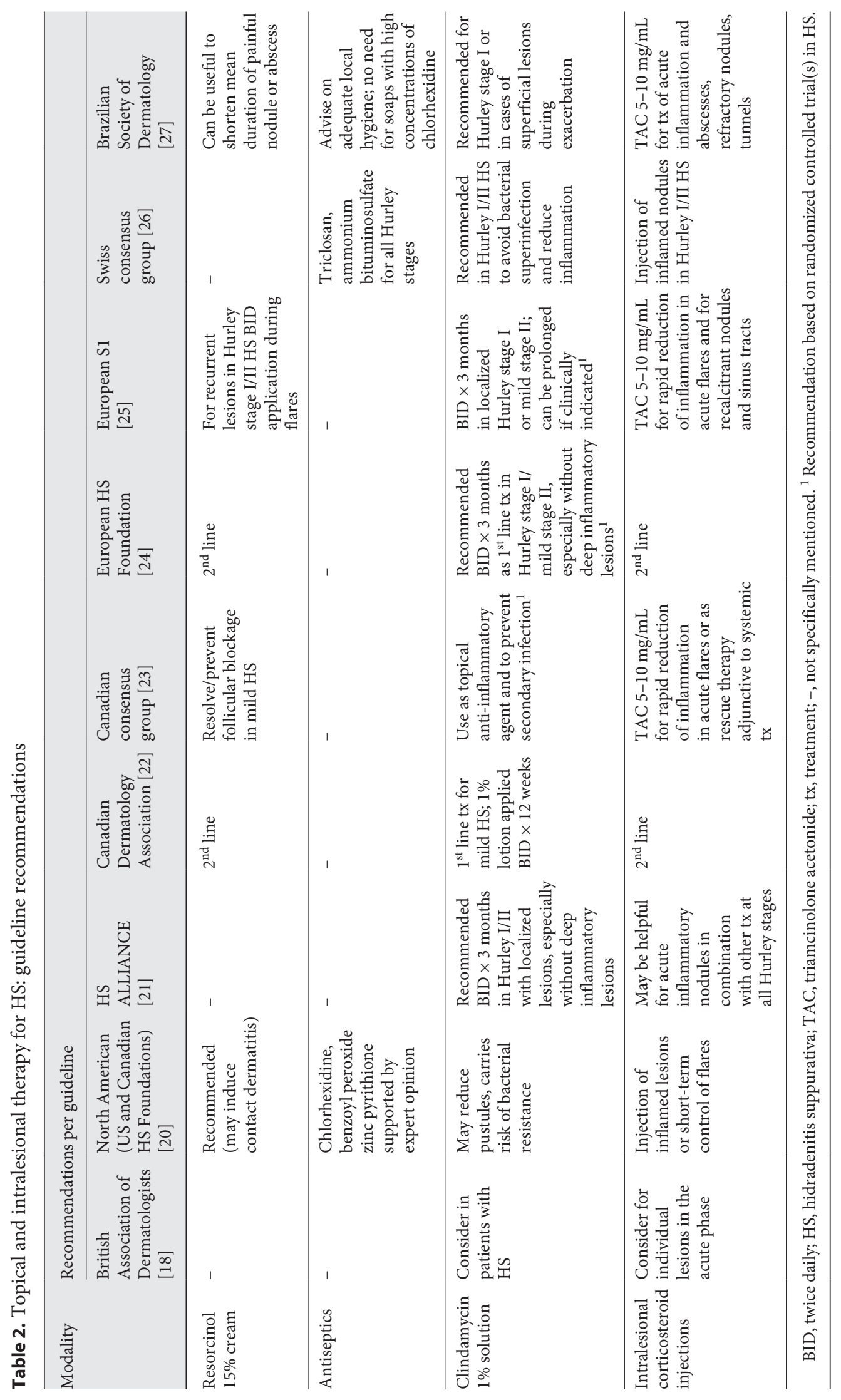


tion triclosan and ammonium bituminosulfonate [26]. Brazilian guidelines do not recommend specific antiseptics, but state that high concentration of chlorhexidine is unnecessary [27]. Availability of these antiseptics varies by region, as the US Food and Drug Administration removed triclosan from the market in December 2018 due to lack of demonstrated efficacy and safety data [32]. Ammonium bituminosulfonate, also known as ichthammol, is a cream or ointment derived from shale oil that is available over-thecounter in both the USA and Europe, but is more commonly recommended for dermatologic use in European medical literature [33].

\section{Topical Antibiotics}

Clindamycin. Clindamycin $1 \%$ topical lotion or solution is recommended across all guidelines as a first-line modality for HS treatment [18, 20-27]. Clindamycin has activity against anaerobic, streptococcal, and staphylococcal species, and plays a role in decreasing cutaneous inflammation and inhibiting biofilm formation in HS [34]. Topical clindamycin has greatest efficacy in less severe disease (Hurley stage I or mild stage II) with predominantly superficial pustules and without deep abscesses. Per the European S1, European HS Foundation, and HS ALLIANCE guidelines, the recommended regimen is twice daily application for up to 3 months [21, 24, $25]$. Several guidelines caution against prolonged use due to the development of bacterial resistance $[20,21,24,25]$.

\section{Intralesional Corticosteroids}

Intralesional corticosteroid injections are recommended by all guidelines as treatment for individual acutely inflamed HS lesions, either as monotherapy or in the setting of concomitant systemic treatment $[18,20$ 27]. Injection of triamcinolone $5-10 \mathrm{mg} / \mathrm{mL}$ (volumes ranging from 0.2 to $2 \mathrm{~mL}$ ) has been shown to decrease size, redness, edema, and suppuration of HS lesions, and provides rapid reduction of patient-reported pain within $1-3$ days $[20,23,35]$. Complications may include skin atrophy and telangiectasias, and intralesional corticosteroid injections are contraindicated if overt bacterial infection is suspected $[25,27]$.

\section{Systemic Therapies}

International guideline recommendations for systemic agents in the treatment of HS are compiled in Table 3.

Systemic Antibiotics

Systemic antibiotics are a mainstay of HS treatment, with mechanisms of action including reduction of in- flammation as well as antibacterial effects. They are key agents in treating widespread disease when application of topical agents is difficult and impractical. Oral tetracyclines are consistently recommended across guidelines as first-line therapy, with a combination regimen of clindamycin and rifampicin as second-line treatment. Third-line options include dapsone or metronidazole/ moxifloxacin/rifampin triple therapy, but limited guidance is provided regarding considerations for selecting a third-line agent. Intravenous ertapenem is reserved for cases refractory to oral antibiotics.

Tetracyclines. Oral tetracycline antibiotics (e.g., doxycycline and minocycline) are recommended for use in mild-to-moderate HS, especially when lesions are widespread or involve multiple anatomical sites. The British, North American, HS ALLIANCE, and Brazilian guidelines suggest a treatment period of 12 weeks $[18,20,21$, 27]. British guidelines recommend holding treatment after 12 weeks to assess the need for continuation and to decrease the likelihood of developing antibiotic resistance [18]. The European HS Foundation and European S1 guidelines recommend oral tetracycline use for up to 4 months [24], while Swiss guidelines suggest a course of 3-6 months [26]. The North American guidelines also discuss long-term use of oral tetracyclines as maintenance therapy [20]. Tetracyclines should be avoided in pregnant women and in children due to the risk of tooth discoloration and interference with bone growth resulting from calcium chelation, although a recent systematic review found that the current literature does not support an association between doxycycline and these potential adverse effects [36].

Clindamycin/Rifampicin. Clindamycin exhibits activity against Streptococci, Staphylococci, and anaerobes, while rifampicin is effective against Gram-positive and intracellular bacterial species. The efficacy of combination therapy is thought to be driven by rifampicin, with clindamycin added to limit rifampicin resistance [37]. A combination regimen of clindamycin and rifampicin is recommended by all guidelines as second-line treatment for mild-tomoderate HS unresponsive to topical agents and oral tetracyclines [18, 20-27]. North American and European HS Foundation guidelines also suggest use as first-line or adjunctive therapy for moderate-to-severe HS [20, 24]. Recommended dosing is clindamycin $300 \mathrm{mg}$ twice daily with rifampicin $300 \mathrm{mg}$ twice daily or $600 \mathrm{mg}$ once daily for a treatment period of 8-12 weeks [18, 20-27]. Brazilian guidelines urge judicious use of rifampicin as it is the firstline medication for the treatment of tuberculosis, which is prevalent in the region with growing multi-agent resis-
86

Dermatology 2021;237:81-96

DOI: $10.1159 / 000503605$
Hendricks/Hsiao/Lowes/Shi 


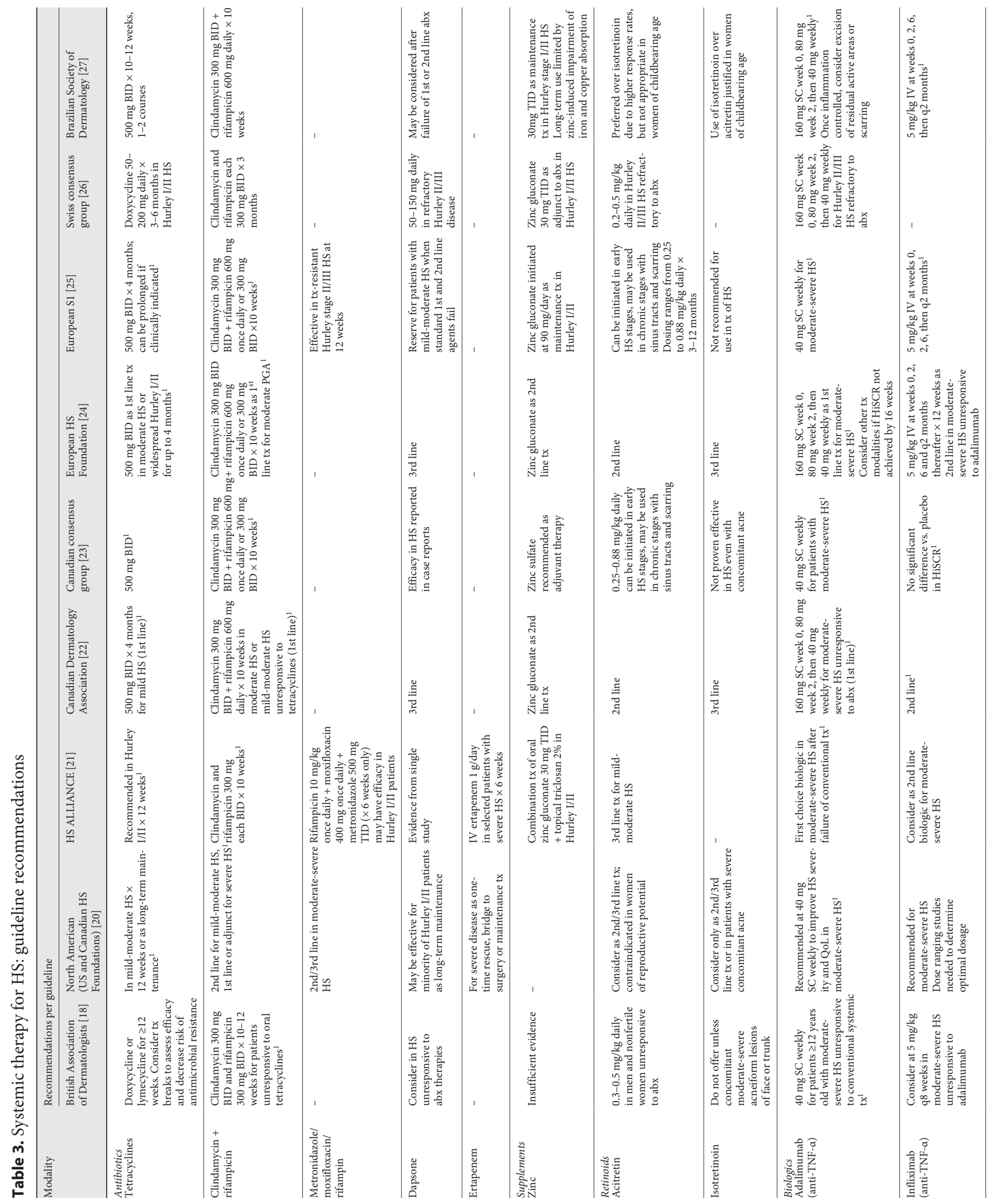




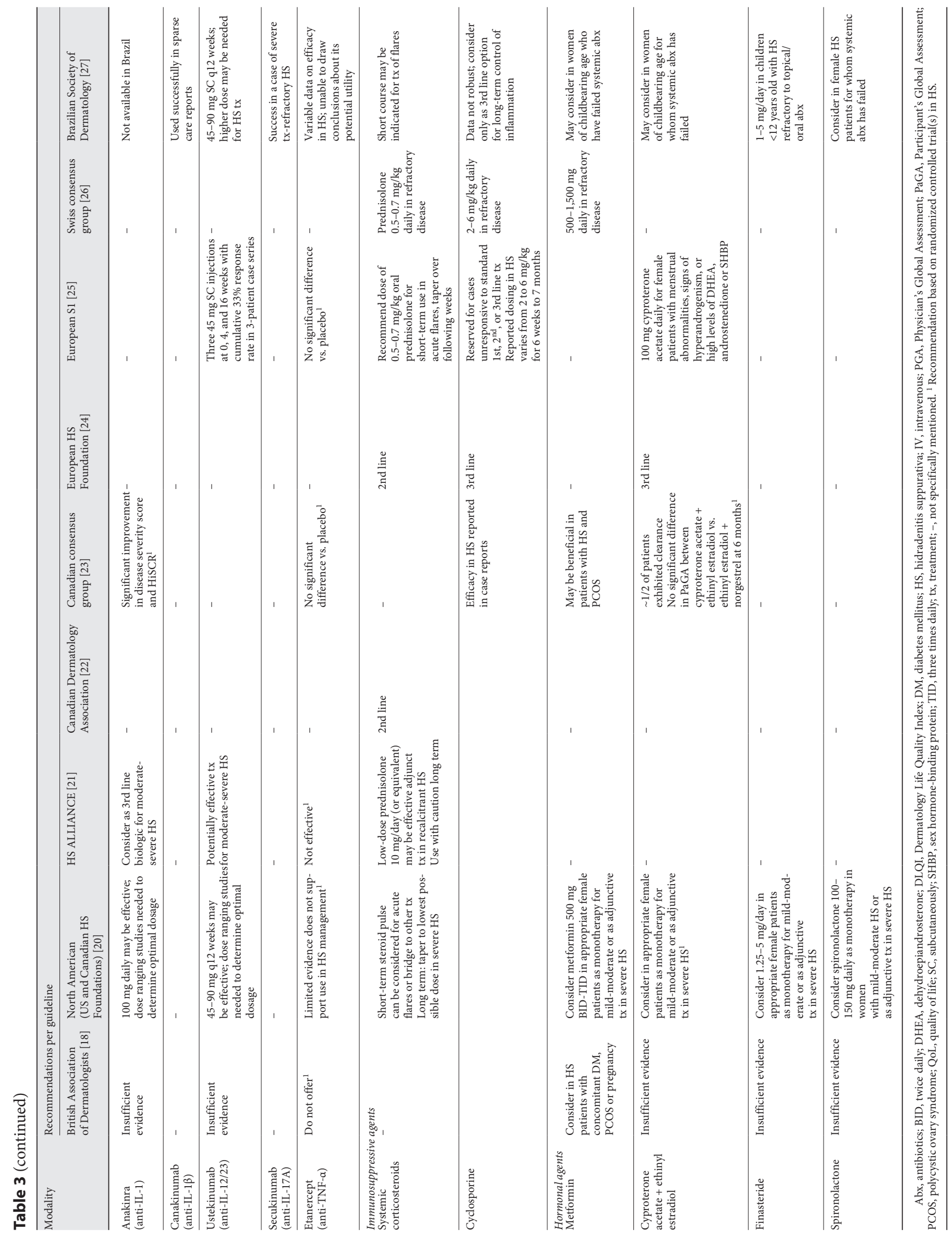


tance [27]. Prolonged use of clindamycin and rifampicin beyond 10 weeks was found to be safe in a recent study [37], but concerns regarding rifampicin resistance should be taken into account when considering extension of therapy [38]. Practitioners should advise patients about redorange discoloration of body fluids with rifampicin and should be vigilant for potential drug-drug interactions, as rifampicin is an inducer of cytochrome P450.

Metronidazole/Moxifloxacin/Rifampin. Triple therapy with moxifloxacin, metronidazole, and rifampin is recommended as second- or third-line treatment in moderate-to-severe HS by North American and European S1 guidelines [20,25], and for use in mild-to-moderate HS by the HS ALLIANCE [21]. Metronidazole is effective against anaerobic bacteria, while moxifloxacin exhibits activity against Gram-positive, Gram-negative, and anaerobic species $[39,40]$. Triple therapy with rifampin provides broad-spectrum coverage and decreases rifampin resistance [40]. Recommended dosing is moxifloxacin $400 \mathrm{mg}$ daily, metronidazole $500 \mathrm{mg} 3$ times a day, and rifampin $300 \mathrm{mg}$ twice daily or $10 \mathrm{mg} / \mathrm{kg}$ once daily [20, 21]. The aforementioned guidelines note that metronidazole should be discontinued after 6 weeks to avoid neurotoxicity [20, 21].

Dapsone. Dapsone is reserved as third-line treatment for HS refractory to other antibiotic regimens, discussed in the British, North American, Canadian Dermatology Association, European HS Foundation, Swiss, and Brazilian guidelines [18, 20, 22, 24, 26, 27]. Dosing ranges from 25 to $200 \mathrm{mg}$ daily with courses of at least 3 months' duration $[20,26,27]$. Prior to initiation of dapsone, glucose6-phosphate dehydrogenase (G6PD) levels should be obtained to assess for G6PD deficiency that could result in hemolytic anemia with administration of sulfa drugs [26].

Ertapenem. Intravenous ertapenem is cited only in the North American and HS ALLIANCE guidelines as an option for HS non-responsive to oral antibiotic therapy [20, 21]. Ertapenem is a broad-spectrum carbapenem antibiotic given daily by an indwelling central catheter with activity against Gram-positive and Gram-negative aerobic and anaerobic species, and exhibits resistance to inactivation by beta-lactamases [41]. Treatment with $1 \mathrm{~g}$ intravenous ertapenem daily led to remission in Hurley stage I/ II disease and significant improvement in quality of life in Hurley stage III patients [42]. Ertapenem is considered as third-line treatment to be used as a one-time 6-week course for rescue therapy or as a bridge to surgical intervention [20], as daily infusions are not feasible for longterm therapy, and peripherally inserted central catheters may increase the risk of infection.

A Comparison of International Management Guidelines for Hidradenitis Suppurativa
Supplements

Zinc. Zinc is thought to play an anti-inflammatory role that may serve as efficacious adjunctive treatment in HS, as low serum zinc levels have been identified in HS patients compared to healthy controls [43]. Zinc has been found to inhibit neutrophil chemotaxis and keratinocyte expression of toll-like receptor 2, both of which are prominent in HS lesional skin [44-47]. The HS ALLIANCE, Canadian Dermatology Association, European HS Foundation, European S1, Swiss, and Brazilian guidelines recommend oral zinc gluconate in mild-to-moderate HS as second-line or adjunctive therapy with topical antiseptics or oral antibiotics [21, 22, 24-27]. Canadian consensus guidelines specifically recommend zinc sulfate rather than gluconate [23]. Four guidelines state recommended dosing, with the HS ALLIANCE, Swiss, and Brazilian guidelines suggesting $30 \mathrm{mg} 3$ times a day $[21,26,27]$ and European $S 1$ guidelines recommending $90 \mathrm{mg}$ daily [25]. Only the Brazilian guidelines mention impaired copper and iron absorption with zinc supplementation, but do not provide specific recommendations for copper and iron replacement [27].

Vitamin D. Vitamin D has been suggested to have a beneficial effect in HS via stimulation of cutaneous innate immunity and modulation of keratinocyte proliferation and hair growth cycling [48-50]. Vitamin D supplementation is mentioned only in the North American guidelines, which cite insufficient evidence to recommend its use [19].

\section{Retinoids}

Retinoids are vitamin A analogs that regulate keratinocyte turnover. Their use in HS is aimed at decreasing follicular keratin plugging that can initiate inflammation in the folliculo-pilosebaceous unit. Acitretin is included across all guidelines as a second- or third-line treatment modality for HS after failure of antibiotics, while the use of isotretinoin is controversial.

Acitretin. Acitretin has demonstrated efficacy in HS and is recommended by all guidelines as second- or thirdline therapy in HS non-responsive to topical and oral antibiotics [18, 20-27]. Suggested dosing ranges from 0.2 to $0.88 \mathrm{mg} / \mathrm{kg}$ daily $[18,23,25,26]$, and is recommended as a second- or third-line agent for use in early or chronic HS. As with all systemic retinoids, reliable contraceptive use is necessary for women of child-bearing potential due to the risk of teratogenicity. British, North American, and Brazilian guidelines state that acitretin is contraindicated in women of childbearing age [18, 20, 27], while European S1 and Swiss guidelines call for close monitoring and routine pregnancy testing in this population $[25,26]$.

Dermatology 2021;237:81-96

DOI: $10.1159 / 000503605$ 
Isotretinoin. Recommendations regarding the use of isotretinoin in HS treatment are conflicting. Canadian consensus and European S1 guidelines recommend against isotretinoin use due to lack of demonstrated efficacy $[23,25]$. British and North American guidelines suggest isotretinoin as a second- or third-line option only for HS patients with moderate-to-severe concomitant acne $[18,20]$. The Canadian Dermatology Association and European HS Foundation recommend isotretinoin only as a third-line treatment option after failure of standard therapies [22, 24]. Brazilian guidelines state that isotretinoin is preferred over acitretin for refractory HS in women of childbearing age [27].

\section{Biologics}

International guidelines recommend consideration of biologics for the treatment of moderate-to-severe HS unresponsive to systemic antibiotics. Signaling molecules targeted by biologics for the treatment of HS include tumor necrosis factor-alpha (TNF- $\alpha$; adalimumab, infliximab), interleukin (IL)-1 (anakinra, canakinumab), IL12/23 (ustekinumab), and IL-17 (secukinumab), which have been shown to be elevated in the skin and serum of HS patients [51-54]. Adalimumab is the only biologic approved for HS and is recommended as the first-line biologic across all guidelines, with infliximab cited as the recommended second-line option in the majority of guidelines. Anakinra and ustekinumab may be considered following failure of anti-TNF agents, but evidence to support their efficacy in HS is limited.

Adalimumab. Adalimumab is a fully-human monoclonal antibody targeting TNF- $\alpha$ and is recommended across all reviewed guidelines as the first-line biologic for Hurley stage II/III HS unresponsive to systemic antibiotics [18, 20-27]. It was approved as the first and only biologic for moderate-to-severe HS in Europe and the USA in 2015 and in Canada in 2016 [55, 56], and its safety and efficacy have been evaluated in randomized controlled trials (RCTs) [57-59]. Adalimumab is administered as a 160 -mg loading dose injected subcutaneously at week 0 , followed by an $80-\mathrm{mg}$ subcutaneous injection at week 2 , and 40-mg subcutaneous injections weekly thereafter. Adverse events associated with anti-TNF- $\alpha$ agents include increased risk of infection [18, 20-27], although long-term studies of TNF- $\alpha$ inhibitors support an encouraging risk-benefit ratio [60].

Infliximab. Infliximab is a chimeric monoclonal antibody against TNF- $\alpha$ that is recommended by all except Swiss and Canadian consensus guidelines as the secondline biologic for moderate-to-severe HS refractory to adalimumab $[18,20-22,24,25,27]$. Although not approved specifically for the treatment of HS, infliximab administered intravenously at $5 \mathrm{mg} / \mathrm{kg}$ at weeks 0,2 , and 6 has been evaluated in an RCT. No significant difference between infliximab and placebo was observed for $>50 \%$ improvement in inflammatory nodules, but the improvement rate of $25-50 \%$ was significantly higher for infliximab compared to placebo [61].

Anakinra. Anakinra is an IL- 1 receptor antagonist that has demonstrated some success in a small RCT involving 20 Hurley stage II/III patients [62]. HS ALLIANCE and North American guidelines note that anakinra may be considered as a third-line treatment option for HS refractory to TNF inhibitors [21], but British and North American guidelines also highlight the need for additional studies to demonstrate efficacy and determine optimal dosing in HS $[18,20]$.

Canakinumab. Canakinumab is a monoclonal antibody targeting IL- $1 \beta$, cited only by the Brazilian guidelines as a third-line biologic for the treatment of HS due to unavailability of anakinra in Brazil [27].

Ustekinumab. Ustekinumab is a monoclonal antibody that modulates IL-12 and IL-23 signaling [63]. In a cohort of 17 patients receiving ustekinumab 45 - or $90-\mathrm{mg}$ (for those weighing $>100 \mathrm{~kg}$ ) subcutaneous injections at weeks $0,4,16$, and $28,82 \%$ achieved at least a $25 \%$ reduction in modified Sartorius score and $47 \%$ achieved HiSCR at week 40 [64]. Based on these findings, ustekinumab is cited as a potentially efficacious HS treatment by the North American, HS ALLIANCE, European S1, and Brazilian guidelines [20,21, 25, 27], although further studies are needed to establish optimal dosing [20].

Secukinumab. Secukinumab is a monoclonal antibody targeting IL-17A and has demonstrated success in a recent pilot study and several case reports of moderate-tosevere HS [65-69]. Secukinumab is cited only in the Brazilian guidelines as a therapeutic consideration for HS that necessitates further evaluation to determine efficacy and HS-specific dosing [27].

Etanercept. Etanercept is a fusion recombinant protein TNF receptor that binds TNF- $\alpha$ with greater affinity than the endogenous receptor, thereby interfering with TNF- $\alpha$ signaling [70]. The British, North American, HS ALLIANCE, Canadian consensus group, and European S1 guidelines recommend against its use in HS treatment $[18,20,21,23,25]$, as a double-blind RCT evaluating etanercept $50-\mathrm{mg}$ subcutaneous injection versus placebo twice weekly in HS patients identified no significant difference between etanercept and placebo after 3 months [70]. Brazilian guidelines state that conclusions about the
Hendricks/Hsiao/Lowes/Shi 
efficacy of etanercept in HS cannot be drawn due to conflicting study results and lack of additional trials [27].

Traditional Immunosuppressive Agents

Broadly immunosuppressive agents such as corticosteroids and cyclosporine are mentioned in the majority of guidelines as third-line therapeutic options for refractory HS. These agents serve to suppress the inflammatory cascade driving HS flares but should be considered for use only after failure of first-line biologics.

Systemic Corticosteroids. Use of systemic corticosteroids in HS management is reserved for short-term courses to treat acute flares or as a bridge to other systemic therapy as cited in the North American, HS ALLIANCE, Canadian Dermatology Association, European HS Foundation, European S1, Swiss, and Brazilian guidelines [2022, 24-27]. North American, European S1, and Swiss guidelines recommend dosing at $0.5-0.7 \mathrm{mg} / \mathrm{kg}$ oral prednisolone with tapering to the lowest possible dose if considered for long-term use $[20,25,26]$. The HS ALLIANCE, Canadian Dermatology Association, European HS Foundation, and Brazilian guidelines do not provide specific dosing recommendations.

Cyclosporine. Cyclosporine is an immunosuppressive agent that inhibits T-cell activation, and is cited as a possible third-line treatment option for HS refractory to standard therapies [20, 22-27]. Although mentioned in the North American, Canadian Dermatology Association, Canadian consensus group, European HS Foundation, European S1, Swiss, and Brazilian guidelines, only the Swiss publication discusses cyclosporine dosing at a proposed regimen of 2-6 $\mathrm{mg} / \mathrm{kg}$ daily [26].

\section{Hormonal Agents}

Hormonal fluctuations and imbalance are thought to contribute to HS pathogenesis, as many female patients report disease flares corresponding to menstrual cycle $[71,72]$. Androgen receptor stimulation also promotes sebum production and follicular occlusion [73], contributing to acneiform-type HS lesions in susceptible individuals. Hormonal regulation may serve as an effective adjunctive treatment in women with comorbidities such as diabetes, polycystic ovary syndrome, or hyperandrogenism. Metformin and antiandrogen therapy are recommended by about half of the guidelines for use in specific subsets of female HS patients.

Metformin. Metformin is cited by the British, North American, Canadian Dermatology Association, Swiss, and Brazilian guidelines for use in specific subgroups of HS patients, including those with concomitant diabetes, polycystic ovary syndrome, or who are pregnant $[18,20$, $23,26,27]$. Recommended dosing according to North American and Swiss guidelines is $500 \mathrm{mg} 2-3$ times daily $[20,26]$, while British, Canadian, and Brazilian guidelines did not specify dosing regimen.

Cyproterone Acetate plus Ethinyl Estradiol. Cyproterone acetate is an antiandrogen agent suggested in the North American, European HS Foundation, European S1, and Brazilian guidelines as monotherapy for female patients with mild-to-moderate HS or those with signs of hyperandrogenism [20, 24, 25, 27]. A randomized double-bind trial compared cyproterone acetate plus ethinyl estradiol versus ethinyl estradiol plus norgestrel contraceptives in 24 female HS patients, and demonstrated clearance of HS lesions in about half of the subjects without a significant difference in response rate between the 2 groups at 6 months. Free androgen index (calculated as total testosterone divided by sex hormone-binding globulin) decreased significantly in both groups, without a difference between the 2 treatment arms [74]. British, HS ALLIANCE, Canadian Dermatology Association, Canadian consensus group, and Swiss guidelines did not address antiandrogen therapy.

Finasteride. Finasteride is a $5 \alpha$-reductase inhibitor that blocks conversion of testosterone to dihydrotestosterone in peripheral tissues and may help to inhibit androgendriven HS exacerbation. Finasteride $1-5 \mathrm{mg}$ daily is discussed as a third-line treatment option in the North American and Brazilian guidelines for use in female or pediatric HS patients, as efficacy has been documented in a limited number of case reports [20,27].

Spironolactone. Spironolactone is an aldosterone receptor antagonist with antiandrogen properties that may be efficacious in female HS patients. Spironolactone is discussed in the North American and Brazilian guidelines, with North American guidelines recommending use as monotherapy for women with mild-to-moderate HS or as adjunctive treatment in severe HS [20], and Brazilian guidelines proposing use in women for whom systemic antibiotics have failed [27]. North American guidelines cite a case series in which spironolactone 100-150 mg daily led to clinical improvement in 17 of 20 female HS patients within 3-6 months of treatment, with 11 of the 20 experiencing complete disease clearance [75].

\section{Procedural Management}

Guideline recommendations for light-based therapies and surgical approaches in HS treatment are summarized in Table 4. Procedural management for HS includes lightbased therapy, lasers, and surgical intervention. Interna- 


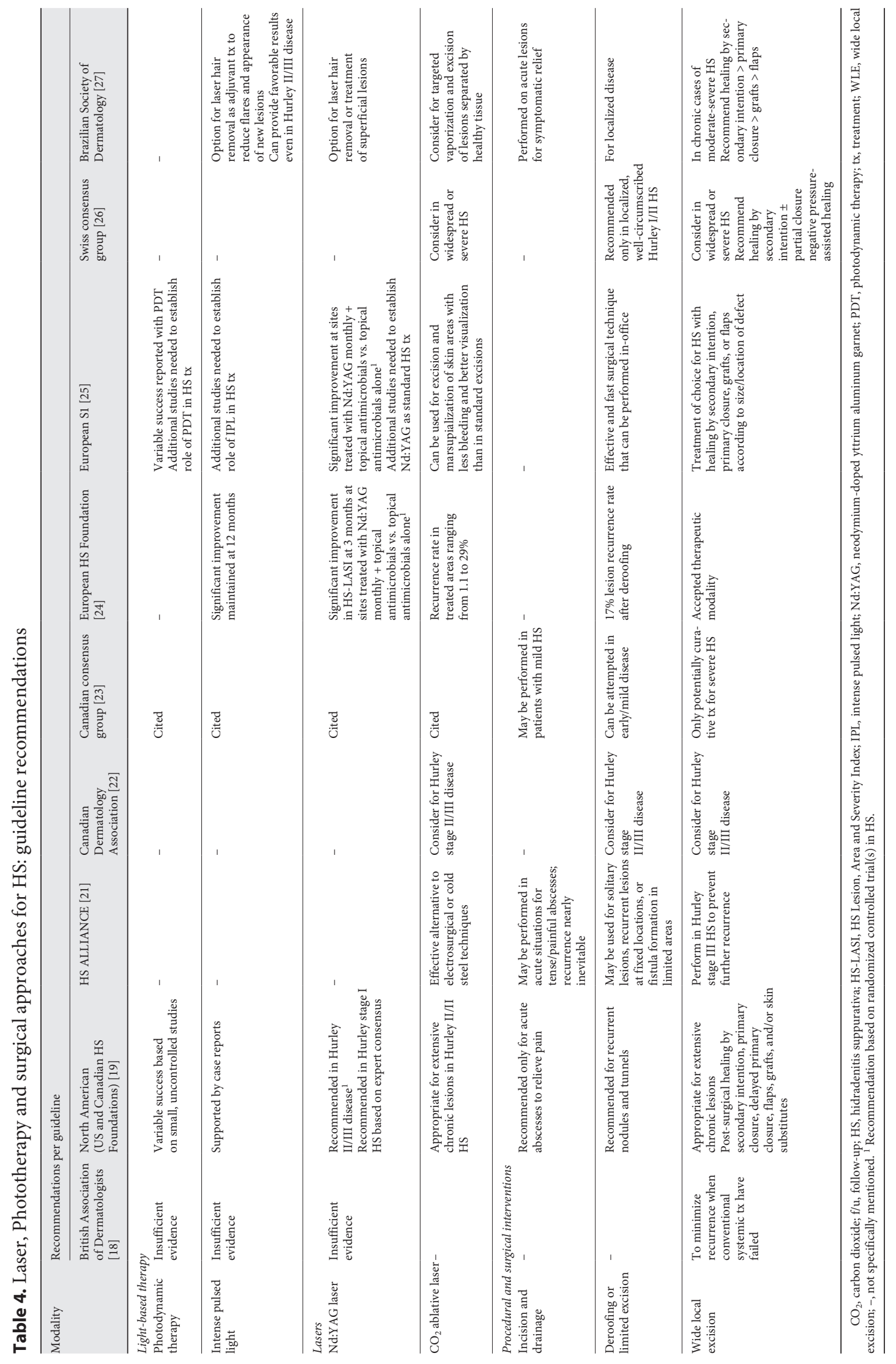


tional recommendations regarding these modalities are somewhat inconsistent, with the exception of carbon dioxide $\left(\mathrm{CO}_{2}\right)$ ablative laser treatment, deroofing, and wide local excision, which are cited as effective interventions in nearly all guidelines. As a general trend, European guidelines tend to be less procedure-intensive compared to North American and Brazilian guidelines.

\section{Light-Based Therapy}

Photodynamic Therapy (PDT). PDT has been used in HS with variable success and is mentioned as a potential treatment option in the North American, Canadian consensus, and European S1 guidelines [19, 23, 25]. PDT utilizing aminolevulinic acid as the sensitizer has yielded variable results in treating HS [76-80]. The HS ALLIANCE, Canadian Dermatology Association, European HS Foundation, and Swiss guidelines make no mention of PDT as a treatment modality for HS, and British guidelines cite insufficient evidence for recommendation. No guidelines mention use of a specific sensitizing agent or distinguish red versus blue light PDT.

Intense Pulsed Light (IPL). IPL for hair reduction is used in HS with the goal of minimizing recurrence. IPL is cited as a potential therapeutic modality by the North American, Canadian consensus, European HS Foundation, European S1, and Brazilian guidelines [19, 23-25, 27], although European $S 1$ and North American guidelines encourage additional research to establish the role of IPL in HS treatment $[18,19,25]$. One split-body RCT demonstrated significant improvement at sites treated twice weekly with IPL compared to untreated sites in Hurley stage II/III subjects [81]. British guidelines cite insufficient evidence to recommend IPL, and the HS ALLIANCE and Canadian Dermatology Association do not discuss IPL as a treatment modality.

\section{Lasers}

Neodymium-Doped Yttrium Aluminum Garnet (Nd:YAG). Nd:YAG laser therapy is recommended by the North American, Canadian Dermatology Association, European HS Foundation, European S1, and Brazilian guidelines for use in Hurley stage II/III disease [19, 23-25, 27], based on an RCT demonstrating significant improvement at sites treated with Nd:YAG and topical antimicrobials compared to topical antimicrobials alone [82]. North American guidelines specifically suggest Nd:YAG as a treatment option for Hurley stage I disease on the basis of expert consensus [19].

$\mathrm{CO}_{2}$ Ablative Laser. $\mathrm{CO}_{2}$ ablative laser therapy is recommended by all except the British guidelines for exten- sive chronic HS lesions in Hurley stage II/III disease [19, 21-27]. The European S1 and Brazilian guidelines note that $\mathrm{CO}_{2}$ laser ablation may be preferable over standard excision due to less bleeding and better visualization of the affected areas with sparing of healthy tissue $[25,27]$.

\section{Procedures}

Incision and Drainage (I\&D). I\&D is recommended in the North American, HS ALLIANCE, Canadian consensus, and Brazilian guidelines for pain relief associated with acute, isolated inflammatory HS lesions [19, 21, 23, 27].

Deroofing. Deroofing is an effective in-office procedure suggested by all except the British guidelines as an intervention for recurrent nodules and sinus tracts, either in localized areas of mild disease or in more extensive Hurley stage II/III HS [19, 21-27].

Wide Local Excision (WLE). WLE is recommended by all guidelines as a surgical intervention to treat advanced regional disease. WLE is reserved for HS refractory to systemic therapy and is recommended for severe but localized disease [18, 19, 21-27]. North American, European S1, Swiss, and Brazilian guidelines discuss that healing after WLE may occur via secondary intention, primary closure, grafts, or flaps according to the size and location of the surgical defect $[19,25-27]$.

\section{Discussion}

Several HS treatment guidelines have emerged since 2015, published by dermatologic organizations and expert working groups in North America, South America, and Europe. While these publications generally share common ground of included studies and case reports, noticeable discrepancies can also be found between recommendations. While there is generally agreement on firstline agents, recommendations for second- and third-line therapeutic options vary significantly and are usually not supported by large-scale high-quality trials. Treatment modalities with strong and uniform support across guidelines include topical clindamycin, oral tetracyclines, combination clindamycin and rifampicin therapy, adalimum$\mathrm{ab}$, and WLE. The majority of these widely supported interventions have been evaluated in RCTs in HS patients, establishing a solid foundation for recommendation and use in regular practice. Although targeted biologics are recommended by several guidelines, issues with insurance coverage are likely to be encountered for those agents not yet indicated for HS in countries without so- 
cialized healthcare systems. Furthermore, a lack of consensus in the use of outcome measurements for HS adds to the variability in assessment of treatment response. This variation could be minimized or potentially solved through the establishment of a core HS outcome set.

All reviewed guidelines place an emphasis on a comprehensive and multidisciplinary approach to treatment of HS patients. This involves patient education, regular communication between specialists, and assessment of HS-related factors such as tobacco use, wound care, depression, and chronic pain. These challenging aspects of day-to-day HS management further highlight the importance of close communication between patients and dermatologists, as well as between dermatologists and providers in the fields of mental health, pain management, and surgery. Complementary and alternative medicine approaches are thoroughly addressed in the North American guidelines [19], but comprehensive information on these modalities is not included in Brazilian, Canadian, or European publications. All reviewed guidelines are put forth by authors from developed regions, but lifestyle modifications and complementary approaches may be of added value in developing nations where medical and surgical interventions are impractical or not readily available.

In an article published recently in the British Journal of Dermatology, Jemec [83] highlights the difficulties encountered in developing guidelines, as new evidence in the field of HS research necessitates regular review and reevaluation of treatment recommendations. There are several targeted agents for HS treatment in the drug development pipeline, further underscoring the importance of timely and comprehensive updates [84]. By coordinating updated guideline publications internationally, experts in HS management can consolidate efforts to publish timely guidelines without duplicating work and with agreement between organizations. The current era repre- sents an exciting time in the treatment of HS with the investigation of new therapeutic modalities and evolution of our understanding of disease pathogenesis. Through international collaboration between dermatologists and with our colleagues in other specialties, we aim to improve the development of comprehensive management guidelines that will lead to optimized care for HS patients.

\section{Key Message}

Some variation exists across international management guidelines for hidradenitis suppurativa. Collaboration between organizations to put forth unified and updated recommendations would be beneficial to HS patients and providers.

\section{Disclosure Statement}

V.Y.S. is a stock shareholder of Learn Health and has served as a consultant or investigator for, or has received research funding from, Sanofi/Regeneron, Eli Lilly, Dermira, Novartis, AbbVie, SUN Pharma, Pfizer, Leo, Menlo Therapeutics, Burt's Bees, BSN, GpSkin, and Skin Actives Scientific unrelated to this paper. M.A.L. has served on the advisory board for AbbVie and Janssen and as a consultant for AbbVie, XBiotech, Incyte, Janssen, BSN, and Almirall unrelated to this paper, and is a member of the Hidradenitis Suppurativa Foundation Medical Board. A.J.H. and J.L.H. have no conflicts of interest to declare relevant to this paper.

\section{Funding Sources}

No funding was received for this study.

\section{Author Contributions}

A.J.H., J.L.H., M.A.L., and V.Y.S. contributed to drafting and revision of the manuscript and approved the final version for submission.

\section{References}

1 Kurzen H, Kurokawa I, Jemec GB, Emtestam L, Sellheyer K, Giamarellos-Bourboulis EJ, et al. What causes hidradenitis suppurativa? Exp Dermatol. 2008 May;17(5): 455-6.

2 Fimmel S, Zouboulis CC. Comorbidities of hidradenitis suppurativa (acne inversa). Dermatoendocrinol. 2010 Jan;2(1):9-16.

3 Saunte DM, Jemec GB. Hidradenitis Suppurativa: Advances in Diagnosis and Treatment. JAMA. 2017 Nov;318(20):2019-32.

4 Garg A, Kirby JS, Lavian J, Lin G, Strunk A. Sex- and Age-Adjusted Population Analysis of Prevalence Estimates for Hidradenitis Suppurativa in the United States. JAMA Dermatol. 2017 Aug;153(8):760-4.

5 Danby FW, Jemec GB, Marsch WC, von Laffert M. Preliminary findings suggest hidradenitis suppurativa may be due to defective follicular support. Br J Dermatol. 2013 May; 168(5):1034-9.

6 Kelly G, Prens EP. Inflammatory Mechanisms in Hidradenitis Suppurativa. Dermatol Clin. 2016 Jan;34(1):51-8.

7 Patel ZS, Hoffman LK, Buse DC, Grinberg AS, Afifi L, Cohen SR, et al. Pain, Psychologi- cal Comorbidities, Disability, and Impaired Quality of Life in Hidradenitis Suppurativa [corrected]. Curr Pain Headache Rep. 2017 Nov;21(12):49.

8 Matusiak $Ł$. Profound consequences of hidradenitis suppurativa: a review. Br J Dermatol. 2018, Epub ahead of print.

9 Vazquez BG, Alikhan A, Weaver AL, Wetter DA, Davis MD. Incidence of hidradenitis suppurativa and associated factors: a population-based study of Olmsted County, Minnesota. J Invest Dermatol. 2013 Jan;133(1):97103 
10 Shavit E, Dreiher J, Freud T, Halevy S, Vinker $\mathrm{S}$, Cohen AD. Psychiatric comorbidities in 3207 patients with hidradenitis suppurativa. J Eur Acad Dermatol Venereol. 2015 Feb;29(2): 371-6.

11 Matusiak L, Bieniek A, Szepietowski JC. Psychophysical aspects of hidradenitis suppurativa. Acta Derm Venereol. 2010 May;90(3): 264-8.

12 Kouris A, Platsidaki E, Christodoulou C, Efstathiou V, Dessinioti C, Tzanetakou V, et al. Quality of Life and Psychosocial Implications in Patients with Hidradenitis Suppurativa. Dermatology. 2016;232(6):687-91

13 Sampogna F, Abeni D, Gieler U, Tomas-Aragones L, Lien L, Titeca G, et al. Impairment of Sexual Life in 3,485 Dermatological Outpatients From a Multicentre Study in 13 European Countries. Acta Derm Venereol. 2017 Apr;97(4):478-82.

14 Thorlacius L, Cohen AD, Gislason GH, Jemec GB, Egeberg A. Increased Suicide Risk in Patients with Hidradenitis Suppurativa. J Invest Dermatol. 2018 Jan;138(1):52-7.

15 Kluger N, Ranta M, Serlachius M. The Burden of Hidradenitis Suppurativa in a Cohort of Patients in Southern Finland: A Pilot Study. Skin Appendage Disord. 2017 Mar;3(1):20-7.

16 Matusiak L, Bieniek A, Szepietowski JC. Hidradenitis suppurativa markedly decreases quality of life and professional activity. J Am Acad Dermatol. 2010 Apr;62(4):706-8.e1.

17 Kirby JS, Miller JJ, Adams DR, Leslie D. Health care utilization patterns and costs for patients with hidradenitis suppurativa. JAMA Dermatol. 2014 Sep;150(9):937-44.

18 Ingram JR, Collier F, Brown D, Burton T, Burton J, Chin MF, et al. British Association of Dermatologists guidelines for the management of hidradenitis suppurativa (acne inversa) 2018. Br J Dermatol. 2019 May;180(5): 1009-1017.

19 Alikhan A, Sayed C, Alavi A, Alhusayen R, Brasasard A, Burkhart C, et al. North American Clinical Management Guidelines for Hidradenitis Suppurativa: a Publication from the United States and Canadian Hidradenitis Suppurativa Foundations: Part I: Diagnosis, Evaluation, and the use of Complementary and Procedural Management. J Am Acad Dermatol. 2019 Jul;81(1):76-90.

20 Alikhan A, Sayed C, Alavi A, Alhusayen R, Brasasard A, Burkhart C, et al. North American Clinical Management Guidelines for Hidradenitis Suppurativa: a Publication from the United States and Canadian Hidradenitis Suppurativa Foundations: Part II: Topical, Intralesional, and Systemic medical Management. J Am Acad Dermatol. 2019 Jul;81(1):91-101.

21 Zouboulis CC, Bechara FG, Dickinson-Blok JL, Gulliver W, Horváth B, Hughes R, et al. Hidradenitis suppurativa/acne inversa: a practical framework for treatment optimization - systematic review and recommendations from the HS ALLIANCE working group. J Eur Acad Dermatol Venereol. 2019 Jan;33(1):19-31.
22 Gulliver W, Landells ID, Morgan D, Pirzada S. Hidradenitis Suppurativa: A Novel Model of Care and an Integrative Strategy to Adopt an Orphan Disease. J Cutan Med Surg. 2018 Jan/Feb;22(1):71-7.

23 Alavi A, Lynde C, Alhusayen R, Bourcier M, Delorme I, George R, et al. Approach to the Management of Patients With Hidradenitis Suppurativa: A Consensus Document. J Cutan Med Surg. 2017 Nov/Dec;21(6):513-24.

24 Gulliver W, Zouboulis CC, Prens E, Jemec GB, Tzellos T. Evidence-based approach to the treatment of hidradenitis suppurativa/ acne inversa, based on the European guidelines for hidradenitis suppurativa. Rev Endocr Metab Disord. 2016 Sep;17(3):34351.

25 Zouboulis CC, Desai N, Emtestam L, Hunger RE, Ioannides D, Juhász I, et al. European S1 guideline for the treatment of hidradenitis suppurativa/acne inversa. J Eur Acad Dermatol Venereol. 2015 Apr;29(4):619-44.

26 Hunger RE, Laffitte E, Läuchli S, Mainetti C, Mühlstädt M, Schiller P, et al. Swiss Practice Recommendations for the Management of Hidradenitis Suppurativa/Acne Inversa. Dermatology. 2017;233(2-3):113-9.

27 Magalhães RF, Rivitti-Machado MC, Duarte GV, Souto R, Nunes DH, Chaves M, et al. Consensus on the treatment of hidradenitis suppurativa - Brazilian Society of Dermatology. An Bras Dermatol. 2019 Apr;94(2 Suppl 1):7-19.

28 Hurley H. Axillary hyperhidrosis, apocrine bromhidrosis, hidradenitis suppurativa, and familial benign pemphigus. In: Roenigk $\mathrm{H}$, Roenigk R, editors. Dermatologic surgery, principles and practice. New York: Marcel Dekker; 1989. pp. 729-39.

29 Dehesa L, Abuchar A, Nuno-Gonzalez A, Vitiello M, Kerdel FA. The use of cyclosporine in dermatology. J Drugs Dermatol. 2012 Aug; 11(8):979-87.

30 Simonart T. Hidradenitis suppurativa and smoking. J Am Acad Dermatol. 2010 Jan; 62(1):149-50.

31 Grond S, Sablotzki A. Clinical pharmacology of tramadol. Clin Pharmacokinet. 2004; 43(13):879-923.

32 U.S. Food and Drug Administration: Q\&A for Consumers: Health Care Antiseptics, 2017.

33 Boyd AS. Ichthammol revisited. Int J Dermatol. 2010 Jul;49(7):757-60.

34 Ring HC, Bay L, Nilsson M, Kallenbach K, Miller IM, Saunte DM, et al. Bacterial biofilm in chronic lesions of hidradenitis suppurativa. Br J Dermatol. 2017 Apr;176(4):993-1000.

35 Riis PT, Boer J, Prens EP, Saunte DM, Deckers IE, Emtestam L, et al. Intralesional triamcinolone for flares of hidradenitis suppurativa (HS): A case series. J Am Acad Dermatol. 2016 Dec;75(6):1151-5.

36 Cross R, Ling C, Day NP, McGready R, Paris $\mathrm{DH}$. Revisiting doxycycline in pregnancy and early childhood-time to rebuild its reputation? Expert Opin Drug Saf. 2016;15(3):36782.
37 Albrecht J, Baine PA, Ladizinski B, Jemec GB, Bigby M. Long-term clinical safety of clindamycin and rifampicin combination for the treatment of hidradenitis suppurativa. A Critically Appraised Topic. Br J Dermatol. 2019 Apr;180(4):749-55.

38 Hambly R, Kirby B. Prolonged clindamycin and rifampicin for hidradenitis suppurativa: resist to prevent resistance. Br J Dermatol. 2019 Apr;180(4):702-3.

39 Caeiro JP, Iannini PB. Moxifloxacin (Avelox): a novel fluoroquinolone with a broad spectrum of activity. Expert Rev Anti Infect Ther. 2003 Oct;1(3):363-70.

40 Join-Lambert O, Coignard H, Jais JP, GuetRevillet H, Poirée S, Fraitag S, et al. Efficacy of rifampin-moxifloxacin-metronidazole combination therapy in hidradenitis suppurativa. Dermatology. 2011 Feb;222(1):4958.

41 Zhanel GG, Johanson C, Embil JM, Noreddin A, Gin A, Vercaigne L, et al. Ertapenem: review of a new carbapenem. Expert Rev Anti Infect Ther. 2005 Feb;3(1):23-39.

42 Join-Lambert $\mathrm{O}$, Coignard-Biehler $\mathrm{H}$, Jais JP, Delage M, Guet-Revillet H, Poirée S, et al. Efficacy of ertapenem in severe hidradenitis suppurativa: a pilot study in a cohort of 30 consecutive patients. J Antimicrob Chemother. 2016 Feb;71(2):513-20.

43 Poveda I, Vilarrasa E, Martorell A, GarcíaMartínez FJ, Segura JM, Hispán P, et al. Serum Zinc Levels in Hidradenitis Suppurativa: A Case-Control Study. Am J Clin Dermatol. 2018 Oct;19(5):771-7.

44 Jarrousse V, Castex-Rizzi N, Khammari A, Charveron M, Dréno B. Zinc salts inhibit in vitro Toll-like receptor 2 surface expression by keratinocytes. Eur J Dermatol. 2007 NovDec;17(6):492-6.

45 Hunger RE, Surovy AM, Hassan AS, Braathen LR, Yawalkar N. Toll-like receptor 2 is highly expressed in lesions of acne inversa and colocalizes with C-type lectin receptor. Br J Dermatol. 2008 Apr;158(4):691-7.

46 Dreno B, Trossaert M, Boiteau HL, Litoux P. Zinc salts effects on granulocyte zinc concentration and chemotaxis in acne patients. Acta Derm Venereol. 1992 Aug;72(4):2502.

47 Lima AL, Karl I, Giner T, Poppe H, Schmidt $\mathrm{M}$, Presser D, et al. Keratinocytes and neutrophils are important sources of proinflammatory molecules in hidradenitis suppurativa. $\mathrm{Br}$ J Dermatol. 2016 Mar;174(3):514-21.

48 Amor KT, Rashid RM, Mirmirani P. Does D matter? The role of vitamin $\mathrm{D}$ in hair disorders and hair follicle cycling. Dermatol Online J. 2010 Feb;16(2):3.

49 Demay MB. The hair cycle and Vitamin D receptor. Arch Biochem Biophys. 2012 Jul; 523(1):19-21.

50 Guillet A, Brocard A, Bach Ngohou K, Graveline N, Leloup AG, Ali D, et al. Verneuil's disease, innate immunity and vitamin $\mathrm{D}$ : a pilot study. J Eur Acad Dermatol Venereol. 2015 Jul;29(7):1347-53.
A Comparison of International

Management Guidelines for Hidradenitis Suppurativa
Dermatology 2021;237:81-96

DOI: $10.1159 / 000503605$ 
51 van der Zee HH, de Ruiter L, van den Broecke DG, Dik WA, Laman JD, Prens EP. Elevated levels of tumour necrosis factor (TNF)- $\alpha$, interleukin (IL)-1 $\beta$ and IL-10 in hidradenitis suppurativa skin: a rationale for targeting TNF- $\alpha$ and IL- $1 \beta$. Br J Dermatol. 2011 Jun; 164(6):1292-8.

52 Witte-Händel E, Wolk K, Tsaousi A, Irmer ML, Mößner R, Shomroni O, et al. The IL-1 Pathway Is Hyperactive in Hidradenitis Suppurativa and Contributes to Skin Infiltration and Destruction. J Invest Dermatol. 2019 Jun; 139(6):1294-305.

53 Thomi R, Cazzaniga S, Seyed Jafari SM, Schlapbach C, Hunger RE. Association of Hidradenitis Suppurativa With T Helper 1/T Helper 17 Phenotypes: A Semantic Map Analysis. JAMA Dermatol. 2018 May;154(5):5925.

54 Matusiak Ł, Szczęch J, Bieniek A, NowickaSuszko D, Szepietowski JC. Increased interleukin (IL)-17 serum levels in patients with hidradenitis suppurativa: implications for treatment with anti-IL-17 agents. J Am Acad Dermatol. 2017 Apr;76(4):670-5.

55 AbbVie. AbbVie's HUMIRA ${ }^{\circledR}$ (Adalimumab) Receives First and Only U.S. Food and Drug Administration Approval for Moderate to Severe Hidradenitis Suppurativa. 2015. Available from: https://news.abbvie.com/ news/abbvies-humira-adalimumab-receivesfirst-and-only-us-food-and-drug-administration-approval-for-moderate-to-severe-hidradenitis-suppurativa.htm

56 AbbVie. AbbVie's HUMIRA ${ }^{\circledR}$ (Adalimumab) Receives First and Only Health Canada Approval for Moderate to Severe Hidradenitis Suppurativa. 2016. Available from: https:// www.newswire.ca/news-releases/abbvies-humira-adalimumab-receives-first-and-onlyhealth-canada-approval-for-moderate-to-severe-hidradenitis-suppurativa-564353901. html.

57 Kimball AB, Kerdel F, Adams D, Mrowietz U, Gelfand JM, Gniadecki R, et al. Adalimumab for the treatment of moderate to severe $\mathrm{Hi}$ dradenitis suppurativa: a parallel randomized trial. Ann Intern Med. 2012 Dec;157(12): 846-55.

58 Kimball AB, Okun MM, Williams DA, Gottlieb AB, Papp KA, Zouboulis CC, et al. Two Phase 3 Trials of Adalimumab for Hidradenitis Suppurativa. N Engl J Med. 2016 Aug; 375(5):422-34.

59 Miller I, Lynggaard CD, Lophaven S, Zachariae C, Dufour DN, Jemec GB. A double-blind placebo-controlled randomized trial of adalimumab in the treatment of hidradenitis suppurativa. Br J Dermatol. 2011 Aug; 165(2): $391-8$.
60 Lim SY, Oon HH. Systematic review of immunomodulatory therapies for hidradenitis suppurativa. Biologics. 2019 May;13:53-78.

61 Grant A, Gonzalez T, Montgomery MO, Cardenas V, Kerdel FA. Infliximab therapy for patients with moderate to severe hidradenitis suppurativa: a randomized, doubleblind, placebo-controlled crossover trial. J Am Acad Dermatol. 2010 Feb;62(2):205-17.

62 Tzanetakou V, Kanni T, Giatrakou S, Katoulis A, Papadavid E, Netea MG, et al. Safety and Efficacy of Anakinra in Severe Hidradenitis Suppurativa: A Randomized Clinical Trial. JAMA Dermatol. 2016 Jan;152(1):52-9.

63 Gulliver WP, Jemec GB, Baker KA. Experience with ustekinumab for the treatment of moderate to severe hidradenitis suppurativa. J Eur Acad Dermatol Venereol. 2012 Jul; 26(7):911-4.

64 Blok JL, Li K, Brodmerkel C, Horvátovich P, Jonkman MF, Horváth B. Ustekinumab in hidradenitis suppurativa: clinical results and a search for potential biomarkers in serum. $\mathrm{Br}$ J Dermatol. 2016 Apr;174(4):839-46.

65 Giuseppe P, Nicola P, Valentina C, Elena C, Salvatrice C, Rosario G, et al. A Case of Moderate Hidradenitis Suppurativa and Psoriasis Treated with Secukinumab. Ann Dermatol. 2018 Aug;30(4):462-4.

66 Jørgensen AR, Yao Y, Thomsen SF. Therapeutic Response to Secukinumab in a 36-YearOld Woman with Hidradenitis Suppurativa. Case Rep Dermatol Med. 2018 Apr;2018: 8685136.

67 Prussick L, Rothstein B, Joshipura D, Saraiya A, Turkowski Y, Abdat R, et al. Open-label, investigator-initiated, single-site exploratory trial evaluating secukinumab, an anti-interleukin-17A monoclonal antibody, for patients with moderate-to-severe hidradenitis suppurativa. Br J Dermatol. 2019 Sep;181(3): 609-11.

68 Schuch A, Fischer T, Boehner A, Biedermann T, Volz T. Successful Treatment of Severe Recalcitrant Hidradenitis Suppurativa with the Interleukin-17A Antibody Secukinumab. Acta Derm Venereol. 2018 Jan;98(1):151-2.

69 Thorlacius L, Theut Riis P, Jemec GB. Severe hidradenitis suppurativa responding to treatment with secukinumab: a case report. $\mathrm{Br} \mathrm{J}$ Dermatol. 2018 Jul;179(1):182-5.

70 Adams DR, Yankura JA, Fogelberg AC, Anderson BE. Treatment of hidradenitis suppurativa with etanercept injection. Arch Dermatol. 2010 May;146(5):501-4.

71 Vossen AR, van Straalen KR, Prens EP, van der Zee HH. Menses and pregnancy affect symptoms in hidradenitis suppurativa: A cross-sectional study. J Am Acad Dermatol. 2017 Jan;76(1):155-6.
72 Riis PT, Ring HC, Themstrup L, Jemec GB. The Role of Androgens and Estrogens in Hidradenitis Suppurativa - A Systematic Review. Acta Dermatovenerol Croat. 2016 Dec;24(4):239-49.

73 Clayton RW, Göbel K, Niessen CM, Paus R, van Steensel MA, Lim X. Homeostasis of the sebaceous gland and mechanisms of acne pathogenesis. Br J Dermatol. 2019 Oct;181(4): 677-90.

74 Mortimer PS, Dawber RP, Gales MA, Moore RA. A double-blind controlled cross-over trial of cyproterone acetate in females with hidradenitis suppurativa. Br J Dermatol. 1986 Sep;115(3):263-8.

75 Lee A, Fischer G. A case series of 20 women with hidradenitis suppurativa treated with spironolactone. Australas J Dermatol. 2015 Aug;56(3):192-6.

76 Gold M, Bridges TM, Bradshaw VL, Boring M. ALA-PDT and blue light therapy for hidradenitis suppurativa. J Drugs Dermatol. 2004 Jan-Feb;3(1 Suppl):S32-5.

77 Saraceno R, Teoli M, Casciello C, Chimenti S. Methyl aminolaevulinate photodynamic therapy for the treatment of hidradenitis suppurativa and pilonidal cysts. Photodermatol Photoimmunol Photomed. 2009 Jun;25(3):164-5.

78 Schweiger ES, Riddle CC, Aires DJ. Treatment of hidradenitis suppurativa by photodynamic therapy with aminolevulinic acid: preliminary results. J Drugs Dermatol. 2011 Apr; 10(4):381-6.

79 Strauss RM, Pollock B, Stables GI, Goulden V, Cunliffe WJ. Photodynamic therapy using aminolaevulinic acid does not lead to clinical improvement in hidradenitis suppurativa. $\mathrm{Br}$ J Dermatol. 2005 Apr;152(4):803-4.

80 Rose RF, Stables GI. Topical photodynamic therapy in the treatment of hidradenitis suppurativa. Photodiagn Photodyn Ther. 2008 Sep;5(3):171-5.

81 Highton L, Chan WY, Khwaja N, Laitung JK. Treatment of hidradenitis suppurativa with intense pulsed light: a prospective study. Plast Reconstr Surg. 2011 Aug;128(2):459-65.

82 Mahmoud BH, Tierney E, Hexsel CL, Pui J, Ozog DM, Hamzavi IH. Prospective controlled clinical and histopathologic study of hidradenitis suppurativa treated with the long-pulsed neodymium:yttrium-aluminium-garnet laser. J Am Acad Dermatol. 2010 Apr;62(4):637-45.

83 Jemec GB. Evidence-based hidradenitis suppurativa guidelines - and now all together please. Br J Dermatol. 2019 May; 180(5):975.

84 Maarouf M, Clark AK, Lee DE, Shi VY. Targeted treatments for hidradenitis suppurativa: a review of the current literature and ongoing clinical trials. J Dermatolog Treat. 2018 Aug;29(5):441-9. 